DOI: http://dx.doi.org/10.14201/0AQ02878596

\title{
GECA: A GLOBAL EDGE COMPUTING ARCHITECTURE
}

\author{
Inés SITTÓN-CANDANEDO
}

BISITE Research Group, University of Salamanca, Edificio Multiusos $I+D+i$, Calle Espejo 2, 37007 Salamanca, Spain

ORCID: 0000-0001-8953-7848

isittonc@usal.es

ABSTRACT: The smart scenarios are in continuous advance thanks to the group of devices that are always connected to Internet. In the last 10 years this phenomenon has been called Internet of Things (IoT). The adoption of the IoT to generate these intelligent scenarios or smart has motivated that governments, universities, research centers or companies are in constant evolution to face the challenges brought by the deployment of IoT platforms. Its disruption in all environments generates large volumes of data, requirements by users for their applications to respond in real time, but with low bandwidth or power consumption, and without delays. The challenges presented by the development of applications for the IoT has occasioned the emergence of technologies such as Edge Computing. This paper presents GECA: A Global Edge Computing Architecture, an architecture based on Edge Computing, which has been deployed in smart farming and smart energy scenarios, with the aim of demonstrating that it is possible to reduce latency, energy consumption and bandwidth costs and integrate Edge computing in IoT platforms.

Keywords: Edge Computing; Reference Architecture; Internet of Things; Industry 4.0; Smart Energy; Smart Farming.

\section{Introduction}

The term Edge Computing is not new, its origin dates back to the 1990s, when Akami Technologies coined it to refer to content delivery networks 
(CDNs) [1]. After Akamai, it is again in 2016 when the term begins to arouse the interest of organizations, governments, universities and researchers for its potential to facilitate the deployment of the Internet of Things.

The Internet of Things, has been defined by various authors as the interaction and communication carried out between the connected devices that generate and exchange data with things or objects of the real world [2-15].

The IoT has extended to a wide variety of environments and disciplines, including solutions for the development of intelligent cities, intelligent farms, smart energy, smart health, logistics and transport. The number of heterogeneous «things» such as buildings, machines, vehicles, homes, people connected to the Internet or to each other through electronic devices that communicate using a set of standard protocols, form a set of networks that can generate, process, store and obtain useful information for organizations. The EC allows the optimization of computing processes as it does not depend on cloud computing for the execution of these processes [16-30].

One of the first scenarios in which the usefulness of Edge Computing has been validated has been Industry 4.0., where it has been presented as an alternative to solve the problems generated by the large data flows generated by IoT devices. In the context of Industry 4.0, A state-of-the-art review have conducted of the main references architectures that include EC technology [31-42].

\section{A Global Edge Computing Architecture}

The revision carried out of the reference architectures that are focus on the use of Edge Computing as a method to reinforce the capabilities of IoT and Cloud Computing, identified a gap in the development of architectures that integrating edge computing, it is possible to deploy in environments other than Industry 4.0. This motivated the design of a new Edge Computing Architecture, with the following purposes:

- Facilitate a real-time analysis of data at the level of local devices and edge nodes, without dependence on the Cloud.

- Reduce operational and management costs by reducing traffic and data transfer between the Edge and the cloud. 
- Improve application performance by achieving lower latency levels at the edge of the network compared to the cloud.

- Enhancing security to through the block chain technologies that will be incorporate to the architecture from the lower layers of IOT to the upper layers of the cloud.

The Global Edge Computing Architecture was proposed by Sittón-Candanedo et al., in [43]. The architecture is made up of three layers (see Figure $1)$ :

- IoT: it is integrated by IoT devices mainly used to monitor services, activities or equipment in operation, such as: sensors, actuators, smartphones, controllers. The communication is done through wireless standards (Wi-Fi, BLE, ZigBee, LoRa or SigFox). The main function of this layer is the management and storage of the computing resources of IoT devices. The security in the architecture starts in this layer by incorporating Blockchain for the protection of the data generated.

- Edge: this layer integrates edge computing for hardware management, represented by the IoT devices of the lower layer. The data filtering and processing processes are carried out in the Edge layer, which, unlike the existing reference architectures, allows the deployment of low-cost solutions such as the micro-controllers incorporated in Raspberry Pi or Orange Pi. The characteristics of these equipment's allow the processing of a much greater amount of data than the ones that are currently incorporated in the IoT devices. The purpose of integrating them into the architecture is to minimize energy consumption and at the same time allow the execution of logic programs and reading control, as well as filtering and preprocessing data using machine learning models and algorithms (e.g.: TensorFlow Lite) on a server based on Node.js that collects data from IoT sensors. Therefore, data filtering running on this layer reduces latency and bandwidth costs by limiting data traffic to the cloud on the platform on which the architecture is deployed [44-50].

- Business Solution Layer: The architecture is designed to integrate the Edge with the Cloud, by including in this third layer the set of services and applications needed today for any business. It can be deployed as an online platform that can run on public or private cloud servers. Calls are made individually to each API and are activated when the user demands 
the use of interactive interfaces of the applications that are part of their business intelligence [50-63].

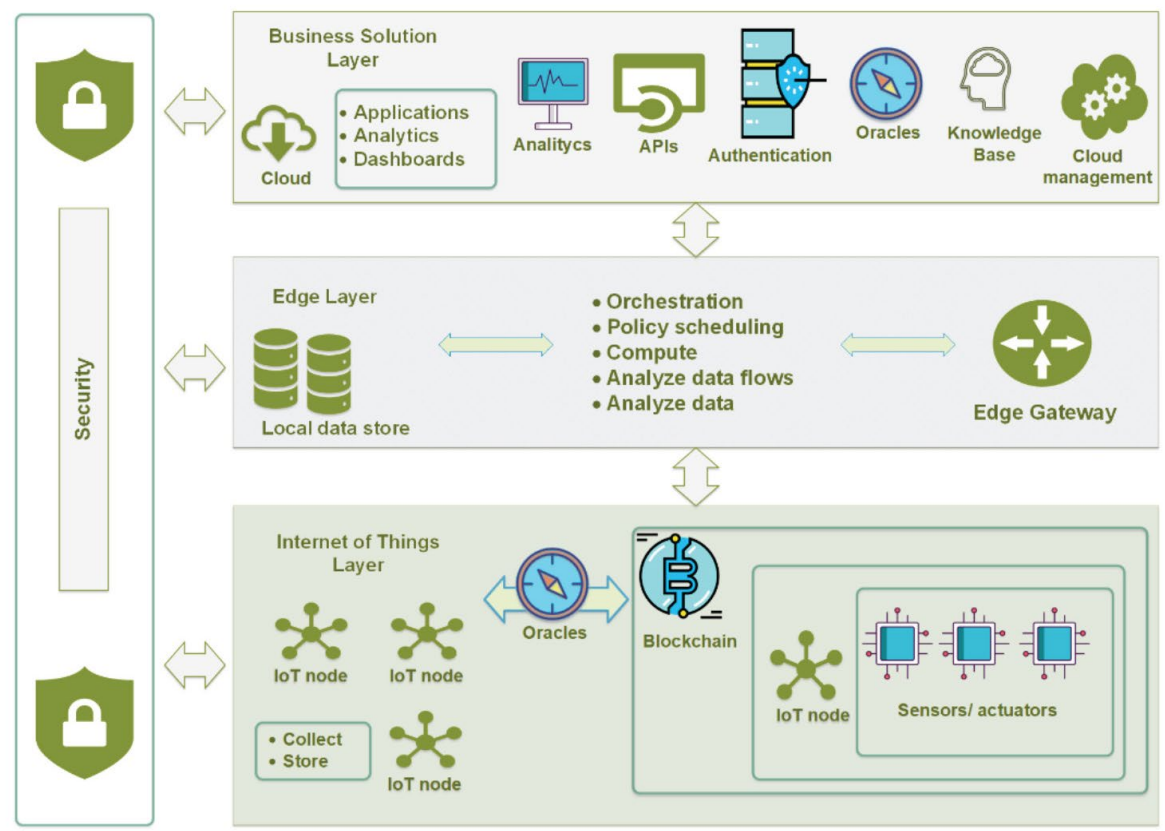

Figure 1. Global Edge Computing Architecture.

So far, the architecture has been deployed in two cases of use:

- Smart Farming: with a platform designed to monitor and optimize the management of agricultural and livestock farms in a mixed dairy farm in Castilla y León, Spain. The aim is to reduce time, bandwidth and storage costs through efficient management. The deployment of GECA made it possible to locate the animals and monitor their health conditions in real time in less time and in a more economical way. On the other hand, the application of the data analytics techniques of the Business Solution layer helped to detect in a timely manner the presence of specific diseases in cows thanks to the association and analysis of the parameters obtained.

- Smart Energy: To validate GECA functionality in a Smart Energy scenario with IoT applications, the architecture was deployed in CAFCLA, a framework to encourage energy consumption efficiency in public 
buildings by applying social computing. CAFCLA was developed and evaluated by [64-70]. When integrated with GECA, two tests of four weeks each were carried out, resulting in a reduction of the data sent to CLOUD. In this scenario the reduction in energy consumption was considerably greater than in Smart Farming [71-75].

To analyze the disruption of Edge Computing in other environments, a state-of-the-art study is conducted to identify existing solutions to the problem posed and present a new solution. In this regard, the Systematic Mapping Study methodology was used to identify existing solutions in which EC is applied to Smart Energy environments [76-80].

The papers analyzed in the SMS were classified according to the type of solutions they offer for a Smart Energy environment. On the other hand, it is a complex scenario with development requirements and proposals aimed at reducing the cost and energy consumption, contributing to obtain operational efficiency, through the intelligent management of infrastructure, make decisions based on data analysis and access to information in real time [81].

\section{Conclusion}

The demand for applications capable of responding in real time regardless of the scenario or the environment variables in which they run is growing.

This demand has contributed to the interruption of increasingly accessible technologies such as large data, the Internet of objects, artificial intelligence, automatic learning models, business intelligence techniques, to mention just a few, which are adopted by large companies to continue operating efficiently and effectively in an increasingly competitive digital market.

Small and medium enterprises seek to access this type of market through cheaper platforms, but at the same time are adequate to ensure their competitiveness and the extraction of the knowledge needed to keep a current company.

This paper presents GECA and its deployment in two smart scenarios in which there are Internet of Things devices. The results obtained in both scenarios demonstrate that it is feasible to provide small organizations or companies with more accessible and economical platforms. GECA allows the reduction of costs for hardware, energy consumption, transfer and storage of 
data to the cloud (whether public or private) and to balance the burden of computing processes.

As future lines of research, GECA will be deployed in other experimental scenarios in which it is also possible to evaluate the reduction of latency, comparison of the reduction of data transfer and costs when using a cloud or another, even evaluating the providers of these services.

\section{References}

1. Akamai Technologies. Technical report, 2007. URL: https://www.akamai.com/es/ es/. Last Access: february 2019.

2. Gil, A. B., De la Prieta, F., \& Rodríguez, S. (2011). Automatic Learning Object Extraction and Classification in Heterogeneous Environments. In Highlights in Practical Applications of Agents and Multiagent Systems (pp. 109-116). Springer, Berlin, Heidelberg.

3. Rodríguez, S., Tapia, D. I., Sanz, E., Zato, C., de la Prieta, F., \& Gil, O. (2010, July). Cloud computing integrated into service-oriented multi-agent architecture. In International Conference on Information Technology for Balanced Automation Systems (pp. 251-259). Springer, Berlin, Heidelberg.

4. Di Mascio, T., Vittorini, P., Gennari, R., Melonio, A., De La Prieta, F., \& Alrifai, M. (2012, July). The Learners' User Classes in the TERENCE Adaptive Learning System. In 2012 IEEE 12th International Conference on Advanced Learning Technologies (pp. 572-576). IEEE.

5. Mazuelas, S., Lorenzo, R. M., Bahillo, A., Fernández, P., Prieto, J., \& Abril, E. J. (2010). Topology Assessment Provided by Weighted Barycentric Parameters in Harsh Environment Wireless Location Systems. IEEE Transactions on Signal Processing, 58(7), 3842-3857. doi:10.1109/TSP.2010.2047394

6. Prieto, J., Alonso, A. A., la Rosa, de, R., \& Carrera, A. (2014). Adaptive Framework for Uncertainy Analysis in Electromagnetic Field Measurements. Radiation Protection Dosimetry. doi:10.1093/rpd/ncu260

7. Prieto, J., Bahillo, A., Mazuelas, S., Blas, J., Fernández, P., \& Lorenzo, R. M. (2008). RTS/CTS mechanism with IEEE 802.11 for indoor location. Presented at the NAV08/ILA37, The Navigation Conference \& Exhibition.

8. Prieto, J., Bahillo, A., Mazuelas, S., Fernández, P., Lorenzo, R. M., \& Abril, E. J. (2012a). Self-Calibration of TOA/Distance Relationship for Wireless Localization in Harsh Environments. Presented at the 2012 IEEE International Conference on Communications (ICC 2012). 
9. Prieto, J., Bahillo, A., Mazuelas, S., Lorenzo, R. M., Blas, J., \& Fernández, P. (2009a). Adding indoor location capabilities to an IEEE 802.11 WLAN using real-time RTT measurements (pp. 113-119). IEEE Press. Retrieved from http:// dl.acm.org/citation.cfm? $\mathrm{id}=1689059.1689083 \&$ coll $=\mathrm{DL} \& \mathrm{dl}=\mathrm{GUIDE} \& \mathrm{CFID}=$ 647274630\&CFTOKEN $=45917612$

10. Bullon, Juan, et al. «Manufacturing processes in the textile industry. Expert Systems for fabrics production.» ADCAIJ: Advances in Distributed Computing and Artificial Intelligence Journal 6.4 (2017): 15-23.

11. Souza de Castro, Lucas Fernando, Gleifer Vaz Alves, \& André Pinz Borges. «Using trust degree for agents in order to assign spots in Smart Parking.» (2017).

12. Moung, Ervin. «A Comparison of the YCBCR Color Space with Gray Scale for Face Recognition for Surveillance Applications.» ADCAIJ: Advances in Distributed Computing and Artificial Intelligence Journal [Online], 6.4 (2017): 25-33.

13. Kethareswaran, V., \& C. SANKAR RAM. «An Indian Perspective on the adverse impact of Internet of Things (IoT).» ADCAIJ: Advances in Distributed Computing and Artificial Intelligence Journal 6.4 (2017): 35-40.

14. Cunha, Rafhael, Cleo Billa, \& Diana Adamatti. «Development of a Graphical Tool to integrate the Prometheus AEOlus methodology and Jason Platform.» ADCAIJ: Advances in Distributed Computing and Artificial Intelligence Journal 6.2 (2017): 57-70.

15. Farias, Giovani Parente, et al. «Predicting Plan Failure by Monitoring Action Sequences and Duration.» ADCAIJ: Advances in Distributed Computing and Artificial Intelligence Journal 6.4 (2017): 55-69.

16. Vera, Jefferson Stewart Espinosa. «Human rights in the ethical protection of youth in social networks-the case of Colombia and Peru.» ADCAIJ: Advances in Distributed Computing and Artificial Intelligence Journal 6.4 (2017): 71-79.

17. Casado-Vara, R., Martin-del Rey, A., Affes, S., Prieto, J., \& Corchado, J. M. (2020). IoT network slicing on virtual layers of homogeneous data for improved algorithm operation in smart buildings. Future Generation Computer Systems, 102, 965-977.

18. Mateen, Abdul, et al. «Secure data access control with perception reasoning.» ADCAIJ: Advances in Distributed Computing and Artificial Intelligence Journal 7.1 (2018): 13-28.

19. Teixeira, Eduardo Porto, Eder Goncalves, \& Diana F. Adamatti. «Ulises: A AgentBased System For Timbre Classification.» ADCAIJ: Advances in Distributed Computing and Artificial Intelligence Journal 7.1 (2018): 29-40.

20. Van Haare Heijmeijer, Alexis, \& Gleifer Vaz Alves. «Development of a Middleware between SUMO simulation tool and JaCaMo framework.» ADCAIJ: Advances in Distributed Computing and Artificial Intelligence Journal 7.2: 5-15. 
21. Glaeser, Stefania da Silveira, et al. «Modeling of Circadian Rhythm under influence of Pain: an approach based on Multi-agent Simulation.» ADCAIJ: Advances in Distributed Computing and Artificial Intelligence Journal 7.2 (2018): 17-25.

22. de Melo, Maximilian Jaderson, et al. «Robust and adaptive chatter free formation control of wheeled mobile robots with uncertainties.» ADCAIJ: Advances in Distributed Computing and Artificial Intelligence Journal 7.2 (2018): 27-42.

23. Ribeiro, Catarina, et al. «Customized normalization clustering meth-odology for consumers with heterogeneous characteristics.» ADCAIJ: Advances in Distributed Computing and Artificial Intelligence Journal 7.2 (2018): 53-69.

24. Becerril, Anahiby Anyel. «The value of our personal data in the Big Data and the Internet of all Things Era.» ADCAIJ: Advances in Distributed Computing and Artificial Intelligence Journal 7.2 (2018): 71-80.

25. Machón-González, I., López-García, H., \& Calvo-Rolle, J. L. (2010, July). A hybrid batch SOM-NG algorithm. In The 2010 international joint conference on neural networks (IJCNN) (pp. 1-5). IEEE.

26. Casado-Vara, R., Novais, P., Gil, A. B., Prieto, J., \& Corchado, J. M. (2019). Distributed continuous-time fault estimation control for multiple devices in IoT networks. IEEE Access, 7, 11972-11984.

27. Garcia, R. F., Rolle, J. L. C., Castelo, J. P., \& Gomez, M. R. (2014). On the monitoring task of solar thermal fluid transfer systems using $\mathrm{NN}$ based models and rule based techniques. Engineering Applications of Artificial Intelligence, 27, 129-136.

28. Rafael Cauê Cardoso, Rafael Heitor Bordini. (2017) A Multi-Agent Extension of a Hierarchical Task Network Planning Formalism. ADCAIJ: Advances in Distributed Computing and Artificial Intelligence Journal (ISSN: 2255-2863), Salamanca, v. 6, n. 2

29. Enyo Gonçalves, Mariela Cortés, Marcos De Oliveira, Nécio Veras, Mário Falcão, Jaelson Castro (2017). An Analysis of Software Agents, Environments and Applications School: Retrospective, Relevance, and Trends. ADCAIJ: Advances in Distributed Computing and Artificial Intelligence Journal (ISSN: 2255-2863), Salamanca, v. 6, n. 2

30. Eduardo Porto Teixeira, Eder M. N. Goncalves, Diana F. Adamatti (2017). Ulises: A Agent-Based System For Timbre Classification. ADCAIJ: Advances in Distributed Computing and Artificial Intelligence Journal (ISSN: 2255-2863), Salamanca, v. 6, n. 2

31. Lucas Fernando Souza de Castro, Gleifer Vaz Alves, André Pinz Borges (2017). Using trust degree for agents in order to assign spots in a Smart Parking. ADCAIJ: Advances in Distributed Computing and Artificial Intelligence Journal (ISSN: 2255-2863), Salamanca, v. 6, n. 2 
32. Aversa, R., Petrescu, R. V., Akash, B., Bucinell, R., Corchado, J., Berto, F., ... \& Petrescu, F. I. (2017). Kinematics and forces to a new model forging manipulator. American Journal of Applied Sciences, 14(1), 60-80.

33. Li, T., Sun, S., Bolić, M., \& Corchado, J. M. (2016). Algorithm design for parallel implementation of the SMC-PHD filter. Signal Processing, 119, 115-127.

34. Corchado, J. M., Aiken, J., Corchado, E. S., \& Fdez-Riverola, F. (2005). Evaluating the air-sea interactions and fluxes using an instance-based reasoning system. AI Communications, 18(4), 247-256.

35. Costa, Â., Novais, P., Corchado, J. M., \& Neves, J. (2011). Increased performance and better patient attendance in an hospital with the use of smart agendas. Logic Journal of IGPL, 20(4), 689-698.

36. Rodríguez, S., de La Prieta, F., Tapia, D. I., \& Corchado, J. M. (2010, June). Agents and computer vision for processing stereoscopic images. In International Conference on Hybrid Artificial Intelligence Systems (pp. 93-100). Springer, Berlin, Heidelberg.

37. Corchado, J. M., Corchado, E. S., \& Pellicer, M. A. (2004, September). Design of cooperative agents for mobile devices. In International Conference on Cooperative Design, Visualization and Engineering (pp. 205-212). Springer, Berlin, Heidelberg.

38. Corchado, J. M., Laza, R., Borrajo, L., Yañez, J. C., De Luis, A., \& Gonzalez-Bedia, M. (2003, July). Agent-based web engineering. In International Conference on Web Engineering (pp. 17-25). Springer, Berlin, Heidelberg.

39. Fdez-Riverola, F., \& Corchado, J. M. (2003). Forecasting red tides using an hybrid neuro-symbolic system. AI Communications, 16(4), 221-233.

40. González-Briones, A., Prieto, J., De La Prieta, F., Herrera-Viedma, E., \& Corchado, J. (2018). Energy optimization using a case-based reasoning strategy. Sensors, 18(3), 865 .

41. Díaz, F., Fdez-Riverola, F., Glez-Peña, D., \& Corchado, J. M. (2006, September). Using fuzzy patterns for gene selection and data reduction on microarray data. In International Conference on Intelligent Data Engineering and Automated Learning (pp. 1087-1094). Springer, Berlin, Heidelberg.

42. Koetsier, J., Corchado, E., MacDonald, D., Corchado, J., \& Fyfe, C. (2004, June). Kernel maximum likelihood hebbian learning. In International Conference on Computational Science (pp. 650-653). Springer, Berlin, Heidelberg.

43. Pavón, J., \& Corchado, J. (2004). Agents for the web. International journal of Web engineering and technology, 1(4), 393-396.

44. Fdez-Riverola, F., Díaz, F., Borrajo, M. L., Yáñez, J. C., \& Corchado, J. M. (2005, August). Improving gene selection in microarray data analysis using fuzzy patterns inside a cbr system. In International Conference on Case-Based Reasoning (pp. 191-205). Springer, Berlin, Heidelberg. 
45. Fernández-Riverola, F., \& Corchado, J. M. (2003, November). Employing tsk fuzzy models to automate the revision stage of a cbr system. In Conference on Technology Transfer (pp. 302-311). Springer, Berlin, Heidelberg.

46. Corchado, J. M., \& Aiken, J. (1998). Expert system for modelling water masses. In WORKSHOP ON DATA MINING. GLASGOW, SCOTLAND.

47. Corchado, J. M., \& Aiken, J. (1998). Neuro-symbolic reasoning for real time oceanographic problems. In CONFERENCE ON DATA MINING. IEE, SAVOY PLACE, LONDON.

48. Corchado, J. M. (1998). Models for integrating artificial intelligence approaches. DOCTORAL CONSORTIUM ON KNOWLEDGE DISCOVERY AND DATA MINING. PAISLEY, UK.

49. Borrajo, M. L., Corchado, J. M., Yáñez, J. C., Fdez-Riverola, F., \& Díaz, F. (2005, August). Autonomous internal control system for small to medium firms. In International Conference on Case-Based Reasoning (pp. 106-121). Springer, Berlin, Heidelberg.

50. Fdez-Riverola, F., Díaz, F., \& Corchado, J. M. (2004, November). Applying rough sets reduction techniques to the construction of a fuzzy rule base for case based reasoning. In Ibero-American Conference on Artificial Intelligence (pp. 83-92). Springer, Berlin, Heidelberg.

51. Corchado, J., \& Lees, B. (1998). Case based reasoning opportunities and technologies. In CONFERENCE ON KNOWLEDGE DISCOVERY. IEE, SAVOY PLACE, LONDON.

52. Corchado, J., \& Lees, B. (1998). Artificial neural networks in pattern recognition: multicollinearity and heterocedasticity. In COLLOQUIUM ON KNOWLEDGE DISCOVERY. LONDON, UK.

53. Corchado, J., \& Lees, B. (1998). An overview of intelligent frameworks. In COLLOQUIUM ON INTELLIGENT SYSTEMS. IEE, LONDON, UK.

54. Corchado, J. M., \& Lees, B. (1998). Probis: Modelling intelligence with hybrid systems. In WORKSHOP ON DATA MINING. University of GLASGOW, SCOTLAND, UK.

55. Corchado, J. (1998). Real time forecast with intelligent systems. In CONFERENCE ON KNOWLEDGE DISCOVERY. IEE, SAVOY PLACE, LONDON.

56. Corchado, J. M. (1997). Bdi multiagent hybrid architecture for project management. In IEEE COLLOQUIUM ON KNOWLEDGE DISCOVERY AND DATA MINING. LONDON ENGLAND.

57. Corchado, J. M. (1997). System for decision making: a practical case. In CONFERENCE ON KNOWLEDGE DISCOVERY AND DATA MINING. IEE, LONDON, UK. 
58. Corchado, J. (1995). Cbr systems, an overview. In INTERNATIONAL CONFERENCE ON INTELLIGENT SYSTEMS. LONDON, ENGLAND, UK.

59. Li, T., Sun, S., Corchado, J. M., \& Siyau, M. F. (2014, July). A particle dyeing approach for track continuity for the SMC-PHD filter. In 17th International Conference on Information Fusion (FUSION) (pp. 1-8). IEEE.

60. Corchado, E. S., Corchado, J. M., Sáiz, L., \& Lara, A. (2004, July). A beta-cooperative cbr system for constructing a business management model. In Industrial Conference on Data Mining (pp. 42-49). Springer, Berlin, Heidelberg.

61. Corchado, J. M., \& Lees, B. (1998). Integration ai models. In WORKSHOP ON KNOWLEDGE DISCOVERY AND DATA MINING. PML-NERC, PLYMOUTHLONDON, UK.

62. Corchado, J. M., \& Lees, B. (1998). Cognitive models for integrating artificial intelligence approaches. In AII WORKSHOP ON KNOWLEDGE DISCOVERY. GLASGOW, UK.

63. Corchado, J. M. (1997). Real time forecast with intelligent systems: Cbrs and anns. In WORKSHOP ON ARTIFICIAL NEURAL NETWORKS. ABERDEEN (pp. $1-3)$.

64. Casado-Vara, R., Prieto, J., De la Prieta, F., \& Corchado, J. M. (2018). How blockchain improves the supply chain: Case study alimentary supply chain. Procedia computer science, 134, 393-398.

65. Corchado, J. M. (1996). Case-base reasoning recommendation system. In IEEE COLLOQUIUM ON KNOWLEDGE DISCOVERY. LONDON, UK.

66. Corchado, J. M. (1995). Neuro-symbolic reasoning-a solution for complex problemas. In INTERNATIONAL CONFERENCE ON INTELLIGENT SYSTEMS. LONDON, UK.

67. Corchado, J. M. (1995). Multi agent tools: a case study. In IEEE COLLOQUIUM ON KNOWLEDGE DISCOVERY. LONDON ENGLAND, UK.

68. Casado-Vara, R., Prieto-Castrillo, F., \& Corchado, J. M. (2018). A game theory approach for cooperative control to improve data quality and false data detection in WSN. International Journal of Robust and Nonlinear Control, 28(16), 5087-5102.

69. Corchado, J. M., \& Lees, B. (2001). Adaptation of cases for case based forecasting with neural network support. In Soft computing in case based reasoning (pp. 293-319). Springer, London.

70. Rodriguez, J. M. C. (2000). Neuro-symbolic model for real-time forecasting problems (Doctoral dissertation, University of Paisley).

71. Casado-Vara, R., Chamoso, P., De la Prieta, F., Prieto, J., \& Corchado, J. M. (2019). Non-linear adaptive closed-loop control system for improved efficiency in IoT-blockchain management. Information Fusion, 49, 227-239. 
72. Li, T., Sun, S., Corchado, J. M., \& Siyau, M. F. (2014, July). Random finite setbased Bayesian filters using magnitude-adaptive target birth intensity. In 17th International Conference on Information Fusion (FUSION) (pp. 1-8). IEEE.

73. Rodríguez, S., Gil, O., De La Prieta, F., Zato, C., Corchado, J. M., Vega, P., \& Francisco, M. (2010, May). People detection and stereoscopic analysis using MAS. In 2010 IEEE 14th International Conference on Intelligent Engineering Systems (pp. 159-164). IEEE.

74. Guillén, J. H., del Rey, A. M., \& Casado-Vara, R. (2019). Security Countermeasures of a SCIRAS Model for Advanced Malware Propagation. IEEE Access, 7, $135472-135478$.

75. Corchado, J. M. (1996). Artificial intelligence models: composed systems as a solution. In IEEE COLLOQUIUM ON KNOWLEDGE DISCOVERY. LONDON ENGLAND, UK.

76. Casado-Vara, R., De la Prieta, F., Rodriguez, S., Prieto, J., \& Corchado, J. M. (2018, June). Cooperative Algorithm to Improve Temperature Control in Recovery Unit of Healthcare Facilities. In International Symposium on Distributed Computing and Artificial Intelligence (pp. 49-62). Springer, Cham.

77. Corchado, J. M. (1995). Hybrid cbr system for real-time temperature forecasting in the ocean. In IEEE COLLOQUIUM ON KNOWLEDGE DISCOVERY. LONDON, UK.

78. Corchado, J. M. (1995). A distributed recommendation system assos. In IEEE COLLOQUIUM ON KNOWLEDGE DISCOVERY. IEE, LONDON, UK.

79. Corchado, J. M. (1995). The use of kernel methods in cbr systems. In INTERNATIONAL CONFERENCE ON INTELLIGENT SYSTEMS. LONDON ENGLAND UK.

80. Corchado, J. M. (1995). Case based reasoning systems: automatic construction. In INTERNATIONAL CONFERENCE ON INTELLIGENT SYSTEMS. LONDON ENGLAND UK.

81. Casado-Vara, R., de la Prieta, F., Prieto, J., \& Corchado, J. M. (2018, November). Blockchain framework for IoT data quality via edge computing. In Proceedings of the 1st Workshop on Blockchain-enabled Networked Sensor Systems (pp. 19-24). ACM. computing in IoT applications, pp. 85-96 